\title{
Non-Obstructive Coronary Artery Disease in Women: Current Evidence and Future Directions
}

Leanna R. Smith, Moro O. Salifu and Isabel M. McFarlane

Department of Internal Medicine, State University of New York, Downstate Health Sciences University, Brooklyn, NY 11203, USA

\section{Abstract}

Background: Over half of women who present with angina are found to have negative coronary angiographic assessments. Of these patients, up to $50 \%$ are diagnosed with coronary microvascular dysfunction (CMD), which refers to pathologic changes within the small vessels of the coronary circulation. The hallmark of the pathophysiology of CMD is that endothelial damage, which occurs due to a multitude of conditions and risk factors, is the inciting event for the development and progression of CMD. CMD leads to a mismatch in myocardial demand and perfusion, leading to signs and symptoms of cardiac ischemia in the absence of obstructive lesions in the major vessels. CMD can be diagnosed through a variety of both invasive methods that allow a more specific evaluation of the microvasculature and non-invasive imaging techniques, such as cardiac positron emission tomography (PET) and magnetic resonance imaging (MRI). Risk factors for CMD overlap significantly with those of obstructive coronary artery disease (CAD) - hypertension, hypercholesterolemia, and diabetes remain salient predictors. However, these conditions only account for $20 \%$ of CMD cases in females.

Findings: Women have sex-specific risk factors such as menopause, pregnancy, polycystic ovarian syndrome (PCOS), and a higher proclivity toward chronic inflammatory disorders. Estrogen has a cardioprotective effect by increasing production of nitric oxide, a potent vasodilator released by endothelial cells. As a result, the hormonal changes of menopause may accelerate endothelial damage, and in turn, CMD. Current treatments focus on addressing the risk factors of cardiovascular disease, such as anti-hypertensive drugs, weight loss, and glucose control.

Conclusion: Given the multifactorial nature of CMD in women, and the extensive atypical risk factors for cardiac disease, a more nuanced approach is needed that addresses the varied pathophysiology of CMD.

\section{Introduction}

Nearly two-thirds of women presenting to the emergency department(ED) with complaining of angina do not have obstructive coronary artery disease on subsequent testing [1]. These patients are discharged after an observation period with the comfort of a normal angiogram. However, 1 out of 13 women will die from a cardiac etiology within the next 10 years, portending a poor prognosis despite a reassuring evaluation [2,3]. In this review, we explore what is known about the diagnosis of coronary microvascular dysfunction (CMD), a condition known to afflict these women presenting with angina with seemingly benign cardiac workups.

CMD is an umbrella term that refers to pathologic changes within small vessels that lead to structural and functional aberrations within coronary microvasculature as they accumulate over time. These abnormal vessels are diminished in their capacity to augment circulation when faced with increased myocardial demand, leading to angina and ischemia [4]. CMD refers to the general principle of these pathological processes that take place, leading to a mismatch in myocardial perfusion and oxygen demand - therefore an exact definition is difficult to pinpoint. Several pathogenic mechanisms are suspected in CMD, such as endothelial cell damage, autonomic nervous system imbalance, smooth muscle dysfunction, and inflammation [5].

Patients with CMD are hospitalized for cardiovascular events at a higher rate than the general population - including for heart failure, sudden cardiac death, and myocardial infarction [6]. They experience a higher incidence of these complications in the setting of negative angiographic assessment. Women suffer from non-obstructive cardiac ischemia at higher rates, and with a greater symptom burden than men [4]. Of these patients, $50 \%$ have evidence of CMD upon further testing [7].

\section{Classification} coronary blood flow [9]. affecting the larger coronary vessels [10]. Isabel.McFarlane@downstate.edu and source are credited.

\section{Publication History:}

Received: October 10, 2020

Accepted: November 22, 2020

Published: November 24, 2020

\section{Keywords:}

Coronary microvascular dysfunction, Microvasculature, Endothelial dysfunction, Endothelial sheer stress, Coronary flow reserve

Though several pathogenic mechanisms have been proposed as the underlying process behind $\mathrm{CMD}$, the prevailing theory focuses on endothelial cell dysfunction as a major cause [4]. Therefore, the main classification scheme splits CMD into endothelial-dependent and endothelial-independent disease [8]. The reason for the dichotomy is that there are different regulatory mechanisms in place to facilitate matching of myocardial perfusion to demand - to ensure that coronary vasculature vasodilates and vasoconstricts according to

Vascular smooth muscle has stretch receptors capable of detecting and responding to intraluminal pressure [9]. Increased pressure signals increased coronary blood flow, and subsequently leads to vasodilation [8]. This represents an endothelial-independent regulatory mechanism, and is most present in medium-sized arterioles. Larger arterioles rely on endothelium-derived cytokines to mediate vasoconstriction and vasodilation, rendering that mechanism endothelium-dependent. Microcirculation is responsible for regulating the overwhelming majority of myocardial perfusion; therefore, significant angina can occur even in the absence of stenosis

"Corresponding Author: Dr. Isabel M. McFarlane, Department of Internal Medicine, State University of New York, Downstate Health Sciences University, Brooklyn, NY 11203, USA, Tel: 718-270-2390, Fax: 718-270-1324; E-mail:

Citation: Smith LR, Salifu MO, McFarlane IM (2020) Non-Obstructive Coronary Artery Disease in Women: Current Evidence and Future Directions. Int J Clin Res Trials 5: 152. doi: https://doi.org/10.15344/2456-8007/2020/152

Copyright: (C) 2020 Smith et al. This is an open-access article distributed under the terms of the Creative Commons Attribution License, which permits unrestricted use, distribution, and reproduction in any medium, provided the original author 
An additional method of classification breaks down CMD into four categories: $\mathrm{CMD}$ without obstructive $\mathrm{CAD}$ or myocardial, $\mathrm{CMD}$ with myocardial disease, $\mathrm{CMD}$ with obstructive $\mathrm{CAD}$, and iatrogenic CMD [4]. Here, we focus on CMD without obstructive CAD.

\section{Pathogenesis}

CMD likely arises from a constellation of aberrant physiologic processes [4]. The causes of ischemia in the absence of obstructive coronary disease have been a point of interest since 1973, a phenomenon termed Cardiac Syndrome X was first written about [11]. The term was introduced in order to describe patients who experienced angina and a positive stress test, but a negative angiographic evaluation [12] The diagnosis is one of exclusion, requiring that cardiac and noncardiac explanations for the chest pain have been explored and ruled out [13]. The incidence of negative angiographic workup was $8 \%$ in men referred for evaluation, and up to $41 \%$ in women [14]. MCD has been proposed as a possible explanation for Cardiac Syndrome X [11]

The subjective experience of pain has also been implicated in Cardiac Syndrome X (CSX), and as a way to explain the variable presentation of patients whose chest pain may be attributed to MCD [15]. Many studies of patients who suffer from CSX do not exhibit objective measures of ischemia upon exposure stimuli that should usually elicit such as with exercise and pharmacological stress testing [16]. Multiple episodes of ischemia resulting from MCD were thought to have the potential to condition cardiac nerve endings, such that they react at a lower threshold to metabolic or inflammatory stimuli [17].

\section{Endothelial Dysfunction}

Coronary endothelial dysfunction is the inciting feature behind development of atherosclerotic plaques [18]. Luminal obstructions can be visualized with a number of techniques, while the functional changes that occur as a result of endothelial damage within the microvasculature cannot be detected [19].

While MCD does not result in significant stenosis on angiographic assessment, it is not a benign condition. Coronary microvascular response to acetylcholine correlates strongly with increased survival, in both obstructive and non-obstructive CAD [20]. Healthy endothelium regulates vascular tone by releasing nitric oxide (NO), a vasodilator, in response to increased myocardial demand [21]. Damage to the endothelium affects the ability of these cells to release $\mathrm{NO}$ in the presence of vasoconstrictor stimuli to maintain myocardial perfusion [20]. The augmentation of the normal endothelial response is net vasoconstriction, leading to the signs and symptoms of myocardial ischemia [22].

While an appropriate supply of $\mathrm{NO}$ is vital to maintaining a match between myocardial perfusion and demand, the balance of vasodilators and vasoconstrictors has also been implicated in CMD [16]. Endothelin-1 is a vasoconstrictor, which opposes the effects of NO. Increased concentrations of endothelin in patients with angina despite normal coronary arteriograms have been observed, clarifying a possible role for abnormal balance of these two endothelial-derived effectors in CMD [23].

There are several clinically-validated means of measuring endothelial function - both invasively and non-invasively. The most reliable method is invasive, and consists of measuring the response of epicardial arteries and microcirculation to a vasodilator, with acetylcholine being used most often [24]. Exercise and the cold pressor test can also be used to induce flow-mediated reactive hyperemia [24]. The cold pressor test involves submerging the subject's hand in ice water, activating the sympathetic nervous system, which should in turn, lead to NO release from functional endothelial cells [25]. In the present of dysfunctional endothelium, NO release will be blunted and the alpha-1 mediated response of vasoconstriction will predominate [25]. Microvasculature responses are evaluated by Doppler or a pressure wire that measures coronary flow reserve (CFR). CFR is a metric commonly employed in clinical studies to assess endothelial function. Where epicardial circulation is concerned, artery diameter can be measured with quantitative coronary angiography or intravascular ultrasound [26].

CFR is a critical metric that is regarded as a stand-in for microvascular function measurement [24]. It refers to the ratio of maximal coronary blood flow ( $\mathrm{CBF}$ ) following stimulation with acetylcholine, adenosine, or exercise, which are all expected to induce vasodilation, to resting CBF. CFR below 2.0 is considered abnormal [4]. The advantage of CFR measurement is that it can quantify both endothelial-dependent, and endothelial-independent function, based on the vasodilator that is used. Acetylcholine will trigger an endothelium-dependent response requiring release of NO. Adenosine reflects more on smooth muscle function, and is therefore endothelium-independent [27].

Non-invasive methods are simpler to deploy as screening tools for an at-risk population. Techniques include use of cardiac MRI and PET to measure CFR, which are discussed in further detail in the section on diagnosis of CMD. These studies are predicated on the fact that the response of the endothelium is critical in inducing reactive hyperemia in the presence of a noxious stimulus, such as ischemia, which can be accomplished with use of a blood pressure cuff inflated tightly to restrict blood flow. Another principle underlying these methods is that measurements of peripheral endothelial function, which are easier to obtain non-invasively, are appropriate surrogates for coronary endothelial function. Therefore, peripheral assessments, such as radial or brachial artery ultrasound, can be employed as well.

One study examined the association with low endothelial sheer stress (ESS) and microvascular endothelial dysfunction, particularly in patients with nonobstructive coronary atherosclerosis [28]. Low ESS refers to the stress created by the friction at the interface of the endothelial surface and flowing blood. It is a hemodynamic phenomenon known to be a pro-inflammatory stimulus, and therefore, a driver of coronary atherosclerosis [29].

Patients who had been referred for angiography following an episode of chest pain were recruited to the study, and were subjected to evaluation of coronary vascular function, both endothelialdependent, and endothelial independent [28]. CBF was calculated before and after administration of acetylcholine, keeping track of the peak change in coronary diameter as a result of acetylcholine and percent change in CBF [28]. These parameters represent epicardial endothelial function and microvascular endothelial function, respectively. The authors found that vessels exhibiting a higher degree of endothelial dysfunction had lower maximal ESS, implicating low ESS, implicating low ESS as a contributing factor for the progression of non-obstructive atherosclerosis [28].

\section{Diagnosis}

Due to the limitations in imaging and histopathological assessment of microvasculature, diagnosis of CMD depends primarily on 
functional assessment, through a variety of modalities [30,31]. More extensive and invasive testing is also available to specifically evaluate the quality of a patient's endothelium if CMD is suspected.

Imaging with cardiac PET can measure myocardial blood flow, which can be employed during rest and stress to approximate CFR. A radioactive tracer allows for continuous monitoring and visualization of vascular defects based on distribution. Cardiac MRI measures myocardial perfusion - patients with CMD have a lower perfusion index than healthy controls. Transthoracic echocardiogram gives comparable results to the more invasive methods of CFR measurement described in the section on endothelial dysfunction.

\section{Clinical Presentation and Risk Factors}

The most common presentation for CMD is angina, although it would not be unusual for a completely asymptomatic presentation [32]. The majority of these patients will not show ischemic changes on electrocardiogram (ECG) [33].

Risk factors for CMD have marked overlap with those of obstructive CAD, such as hypertension, diabetes, and hyperlipidemia. Hypertension and hyperlipidemia contribute to the development of obstructive CAD, and these risk factors have proven to correlate more strongly with disease progression in men than women [34]. However, diabetes and tobacco use confer a higher risk for CAD in women than men [35,36]. Given that many hallmarks of diabetes involve microvascular dysfunction, we expect a similar pathologic process for the coronary microvasculature [37]. The prevalence of CMD among women compared to male patients may provide a reason for the discrepancy in how the risk factor affects each sex. Chronic hyperglycemia negatively affects both endothelial-dependent and independent vasodilation, both of which are hallmarks in pathogenesis of CMD [38]. See Figure 1.
Chronic inflammatory conditions also pose greater risk of cardiovascular disease - patients with rheumatoid arthritis face a 1.5 fold higher risk than the general population [39]. One study looked at the incidence of decreased CFR, considered a surrogate for microvascular function among rheumatoid arthritis (RA) and diabetes mellitus (DM) patients. They observed that the CMD burden, approximated by CFR obtained from stress myocardial perfusion PET scans was similar in both groups of patients. The authors concluded that their data suggests a cause and effect relationship between persistent inflammation present in RA and CMD. Prior studies have shown an association between higher levels of RA disease burden and markers of endothelial dysfunction, such as flow mediated dilatation $[40,41]$. Females were overrepresented in the RA arm of the study, which is consistent with RA predominance in women in the general population, identifying the chronic inflammatory state of RA as a risk factor that preferentially affects women. Other studies have positively correlated systemic lupus erythematous with increased rates of CMD compared with control subjects [42].

The WISE study found that under $20 \%$ of cases of non-obstructive CAD in women could be accounted for by the typical risk factors associated with CAD [43]. The prevalence of CMD among women, in the absence of classic predisposing factors, suggests that other, sex-specific factors are at play in determining the initiation and progression of CMD in women [44]. The WISE study also discovered that CMD was most common in women in perimenopause and menopause, highlighting estrogen deficiency as a possible risk factor for CMD [45].

Another salient risk factor for development of CMD is aging. Aging is associated with many physiologic changes that affect hemodynamic parameters, namely increased arterial stiffness and thickening, as well as remodeling that all contribute to lower CFR [46].

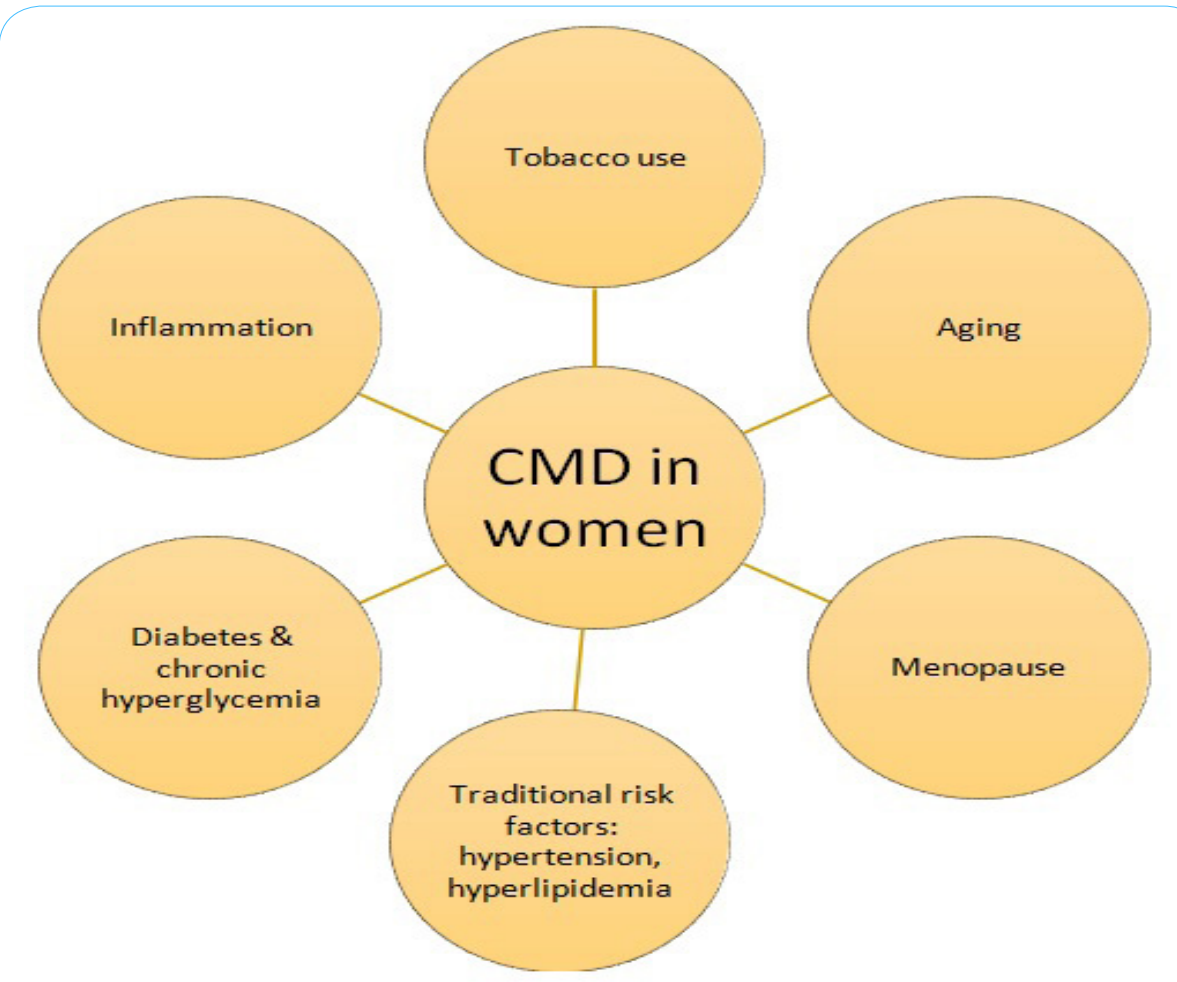

Figure 1: Risk factors for development and progression of coronary microvascular dysfunction in women. 


\section{Sex Differences}

Aging has been implicated as a culprit behind endothelial dysfunction - while both men and women age, CMD is more prevalent in middle-aged women than men. There is overwhelming consensus in the literature that women present with angina despite normal coronaries at a higher rate than men. The WISE study indicated that microvascular dysfunction produced the signs and symptoms of angina in women [47].

There are also differences in pathophysiology between males and females to account for, as well as life cycle events like menopause and pregnancy, in addition to disease states such as preeclampsia, gestational diabetes, PCOS, and inflammatory disorders, that women experience at a higher rate than men. Women typically begin to experience endothelial dysfunction in the period leading up to menopause, and continue to experience a decline in the postmenopausal period [48]. For most women, these transitions are extrinsically linked to age. Studies have shown that women who have had oophorectomy exhibit endothelial dysfunction as evidenced by blunted endothelial dependent dilation. More severe symptoms of menopause may also be related specifically, vasomotor symptoms, which studies have linked to subclinical atherosclerosis [49]. Severe episodes of vasomotor symptoms involve increased sympathetic activation, and decreased parasympathetic activation, which can speed progression of endothelial dysfunction, as well as disturb an already delicate balance of vasoconstrictor and vasodilator stimuli [48]. Estrogen is thought to have a protective effect on the endothelium by inducing nitric oxide release, and the drop in circulating hormone during this transition is also thought to allow endothelial dysfunction and CMD to proceed more quickly.

Pregnancy is a high-output state characterized by increased maternal blood volume that is known to alter several hemodynamic parameters. As a result, pregnancy may unmask previously subclinical cardiovascular disease, or identify women who are at higher risk later in their lives. Up to $10 \%$ of pregnancies are affected by hypertensive disorders [48].

Several studies have shown that pregnant patients who develop preeclampsia have decreased capacity for vasodilation, which is largely a function of intact endothelium. The hypertensive component of preeclampsia is thought to be the result of increased blood volume without the resulting decreased in systemic vascular resistance, which would be facilitated by vasodilation [50]. Several studies point to $\mathrm{NO}$ as a culprit in defective vasodilation in preeclampsia with endothelial cells of non-preeclamptic women producing more NO than endothelium from individuals experiencing preeclampsia [51]. Some researchers characterize preeclampsia as a chronic inflammatory state, in which TNF-alpha is elevated, which affects endothelial cells by decreasing responsiveness to vasodilator stimuli [52]. While preeclampsia is pathologic condition of pregnancy, the endothelium can sustain long-term damage. One study posits that prolonged exposure of the endothelium to inflammatory cytokines and growth factors can affect the endothelial monolayer by causing cell junction proteins to degrade and retract, leading to increased vascular permeability and endothelial dysfunction [53]. This proposed mechanism makes sense in the context of preeclampsia - one of the condition's hallmarks is proteinuria, resulting from leaky glomerular endothelial cells. In addition, endothelial damage incurred during pregnancy, particularly in the ovarian circulation, has been linked to ovarian insufficiency, lessening the protective effects afforded by premenopausal levels of circulating estrogen [53]. Chronic hypertension risk is significant elevated in women who experience preeclampsia - a meta-analysis found such patients to be four times more likely to receive a hypertension diagnosis, which is a typical risk factor for development of both obstructive and non-obstructive CAD, and particularly salient in endothelial dysfunction and CMD progression [54]. Preeclampsia is recognized as a serious disorder of pregnancy, but its prolonged effects on women's vascular health deserves attention as well.

Gestational diabetes mellitus (GDM) can be similarly linked to dysfunction in maternal circulation. The combination of insulin resistance, as well as the derangement of coagulation and inflammatory factors, is suspected to contribute to GDM leading to an increased risk profile [55]. Vascular endothelial dysfunction, and therefore CMD, become more likely, much in the same way that non-pregnancy related diabetes induces changes in the microcirculation. The Cardiovascular Health After Maternal Placental Syndromes (CHAMPS) study, which retrospectively analyzed the records of 1 million women, concluded that women with any gestational complication were twice as likely to experience CVD in the 90 days following delivery compared with patients who had uneventful pregnancies [48]. GDM and hypertensive disorders of pregnancy present a valuable opportunity to address future CVD risk and potential risk factor modification and mitigation.

A study of 50 healthy men and women was configured to assess their coronary endothelial function with cardiac MRI in response to an endothelial-dependent stressor. When the results were stratified based on age and sex, the authors concluded that premenopausal women had superior coronary endothelial function by nearly twofold, leading them to believe that a fundamental age difference was relevant in the development of CMD [56]. Further strengthening the connection between menopause and endothelial dysfunction is the face that early onset ovarian dysfunction, which affects up to $1 \%$ of women increases risk for ischemic heart disease [57]. While this idea validates a relationship where ovarian insufficiency predates endothelial dysfunction, and therefore CMD, some researchers have also posited that women who experience vascular dysfunction may experience menopause at a younger age due to compromise of the ovarian blood supply [58]. In these instances, ovarian insufficiency resulting from vascular damage would further compound the hormonal deficiencies known to contribute to the progression of MCD in women [59].

\section{Conclusion}

The prevalence of MCD in women compared to men suggests the need to target the microcirculation early on in atherogenesis, specifically when women present with cardiac angina in the absence of luminal obstruction. However, cardiovascular disease is the leading cause of morbidity and mortality for women. Many risk factors that are unique to women have been identified that help to explain the predominance of CMD in women, beyond the typical risk factors for atherosclerosis, which only account for a minority of CMD cases.

Current treatment paradigms focus on mainstays in cardiovascular disease and on modifiable risk factors, such as weight loss and regular physical activity. Due to the multifactorial nature of CMD pathogenesis, a multipronged approach is needed to treat patients, depending on the pathologic features of the disease. Since estrogen deficiency is a risk factor for CMD development, it has potential as anantianginal treatment, but is currently contraindicated as a preventive measure for cardiovascular disease [60]. 
Citation: Smith LR, Salifu MO, McFarlane IM (2020) Non-Obstructive Coronary Artery Disease in Women: Current Evidence and Future Directions. Int J Clin Res Trials 5: 152. doi: https://doi.org/10.15344/2456-8007/2020/152

Page 5 of 6

However, for women with primary ovarian insufficiency, hormonal therapy is currently recommended until they reach the average age of menopause.

Given that there is little consensus on the appropriate treatment for $\mathrm{CMD}$, this gives further credence to the necessity of representation of women in clinical trials. Further studies are needed to clarify why aging seems to selectively affect the microvasculature of women, and whether there is a connection between the hormonal changes typical of menopause and endothelial dysfunction. In addition, further research is necessary to establish a mechanistic relationship between GDM and hypertensive diseases of pregnancy and endothelial dysfunction, and subsequent CMD, as such information could guide early interventions and monitoring initiatives for otherwise healthy, mostly young women, who are at increased risk for ischemic heart disease later in life.

\section{Competing Interests}

The authors declare that they have no competing interests.

\section{Acknowledgement}

This work is supported in part by Dr. Moro O. Salifu's efforts through NIH Grant \# S21MD012474

\section{References}

1. BaireyMerz CN, Pepine CJ, Walsh MN, Fleg JL (2017) Ischemia and no obstructive coronary artery disease (INOCA): developing evidence-based therapies and research agenda for the next decade. Circulation 135: 10751092.

2. Kenkre TS, Malhotra P, Johnson BD, Handberg EH, Thompson DV, et al (2017) Ten-Year Mortality in the WISE Study (Women's Ischemia Syndrome Evaluation). Circ Cardiovasc Qual Outcomes 10: e003863.

3. Jespersen L, Hvelplund A, Abildstrøm SZ, Pedersen F, Galatius S, et al (2012) Stable angina pectoris with no obstructive coronary artery disease is associated with increased risks of major adverse cardiovascular events. Eur Heart J 33: 734-744.

4. Camici PG, Crea F (2007) Coronary microvascular dysfunction. N Engl J Med 356: 830-840.

5. Kothawade K, BaireyMerz CN (2011) Microvascular coronary dysfunction in women: pathophysiology, diagnosis, and management. Curr Probl Cardiol 36: 291-318.

6. Pepine CJ, Anderson RD, Sharaf BL, Reis SE, Smith KM, et al. (2010) Coronary microvascular reactivity to adenosine predicts adverse outcome in women evaluated for suspected ischemia: Results from the National Heart, Lung and Blood Institute WISE (Women's Ischemia Syndrome Evaluation) study. Am Coll Cardiol 55: 2825-2832.

7. Sharaf BL, Pepine CJ, Kerensky RA, Reis SE, Reichek N, et al. (2001) Detailed angiographic analysis of women with suspected ischemic chest pain (pilot phase data from the NHLBI-sponsored Women's Ischemia Syndrome Evaluation [WISE] Study Angiographic Core Laboratory). Am J Cardiol 87 937-941.

8. Chen C, Wei J, AlBadri A, Zarrini P, Merz NB, et al. (2017) Coronary Microvascular Dysfunction - Epidemiology, Pathogenesis, Prognosis, Diagnosis, Risk Factors and Therapy. Circ J 81: 3-11.

9. Kuo L, Davis MJ, Chilian WM (1995) Longitudinal gradients for endotheliumdependent and -independent vascular responses in the coronary microcirculation. Circulation 92: 518-525.

10. Ohman EM (2016) Chronic stable angina. N Engl J Med 375: 293

11. Kemp HG Jr, Vokonas PS, Cohn PF, Gorlin R (1973) The anginal syndrome associated with normal coronary arteriograms. Report of a six year experience. Am J Med 54: 735-742.

12. Cannon RO 3rd, Epstein SE (1988) Microvascular "angina" as a cause of chest pain with angiographically normal coronary arteries. Am J Cardiol 61: 1338-1343.
13. Kaski JC, Crea F, Nihoyannopoulos P, Hackett D, Maseri A, et al. (1986) Transient myocardial ischemia during daily life in patients with syndrome. Am J Cardiol 58: 1242-1247.

14. Sullivan AK, Holdright DR, Wright CA, Sparrow JL, Cunningham D, et al. (1994) Chest pain in women: clinical, investigative, and prognostic features. BMJ 308: 883-886.

15. Pasceri V, Lanza GA, Buffon A, Montenero AS, Crea F, et al. (1998) Role of abnormal pain sensitivity and behavioral factors in determining chest pain in syndrome. J Am CollCardiol 31: 62-66.

16. Kothawade K, Merz CNB (2011) Microvascular Coronary Dysfunction in Women - Pathophysiology, Diagnosis, and Management. Curr Probl Cardiol 36: 291-318.

17. Crea F, Lanza GA (2004) Angina pectoris and normal coronary arteries: cardiac syndrome. Heart 90: 457-463.

18. Gutiérrez E, Flammer AJ, Lerman LO, Elízaga J, Lerman A, et al. (2013) Endothelial dysfunction over the course of coronary artery disease. Eur Heart J 34: 3175-3181.

19. Jespersen L, Hvelplund A, Abildstrøm SZ, Pedersen F, Galatius S, et al. (2012) Stable angina pectoris with no obstructive coronary artery disease is associated with increased risks of major adverse cardiovascular events. Eur Heart J 33: 734-744.

20. Halcox JP, Schenke WH, Zalos G, Mincemoyer R, Prasad A, et al. (2002) Prognostic value of coronary vascular endothelial dysfunction. Circulation 106: 653-658.

21. Sodha NR, Boodhwani M, Clements RT, Feng J, Xu SH, et al. (2008) Coronary microvascular dysfunction in the setting of chronic ischemia is independent of arginase activity. Microvasc Res 75: 238-246.

22. Bugiardini R, Badimon L, Collins P, Erbel R, Fox K, et al. (2007) Angina, "normal" coronary angiography, and vascular dysfunction: risk assessment strategies. PLoS Med 4: e12.

23. Kaski JC, Elliott PM, Salomone O, Dickinson K, Gordon D, et al. Concentration of circulating plasma endothelin in patients with angina and normal coronary angiograms. Br Heart J 74: 620-624.

24. Hess OM, Büchi M, Kirkeeide R, Niederer P, Anliker M, et al. (1990) Potential role of coronary vasoconstriction in ischaemic heart disease: effect of exercise. Eur Heart J. 11: 58-64.

25. Nabel EG, Ganz P, Gordon JB, Alexander RW, Selwyn AP, et al. (1988) Dilation of normal and constriction of atherosclerotic coronary arteries caused by the cold pressor test. Circulation 77: 43-52.

26. Flammer AJ, Anderson T, Celermajer DS, Creager MA, Deanfield J, et al. (2012) The assessment of endothelial function: from research into clinical practice. Circulation 1260: 753-767.

27. Kothawade K, BaireyMerz CN (2011) Microvascular coronary dysfunction in women: pathophysiology, diagnosis, and management. Curr Probl Cardiol 36: 291-318.

28. Siasos G, Sara JD, Zaromytidou M, Park KH, Coskun AU, et al. (2018) Local Low Shear Stress and Endothelial Dysfunction in Patients With Nonobstructive Coronary Atherosclerosis. J Am Coll Cardiol 71: 2092-2102.

29. Stone PH, Coskun AU, Kinlay S, Clark ME, Sonka M, et al. (2003) Effect of endothelial shear stress on the progression of coronary artery disease, vascular remodeling, and in-stent restenosis in humans: in vivo 6-month follow-up study. Circulation 108: 438-444.

30. Beltrame JF, Crea F, Camici P (2009) Advances in coronary microvascular dysfunction. Heart Lung Circ 18: 19-27.

31. Radico F, Cicchitti V, Zimarino M, Caterina RD (2014) Angina pectoris and myocardial ischemia in the absence of obstructive coronary artery disease: practical considerations for diagnostic tests. JACC Cardiovasc Interv 7: 453463.

32. Taqueti VR, Di Carli MF (2018) Coronary Microvascular Disease Pathogenic Mechanisms and Therapeutic Options. J Am Coll Cardiol 72: 2625-2641.

33. Epstein SE, Cannon RO, Bonow RO (1991) Exercise testing in patients with microvascular angina. Circulation 83: III73-III76.

34. Suessenbacher A, Wanitschek M, Dörler J, Neururer S, Frick M, et al. (2014) Sex differences in independent factors associated with coronary artery disease. Wien Klin Wochenschr 126: 718-726. 
Citation: Smith LR, Salifu MO, McFarlane IM (2020) Non-Obstructive Coronary Artery Disease in Women: Current Evidence and Future Directions. Int J Clin Res Trials 5: 152. doi: https://doi.org/10.15344/2456-8007/2020/152

Page 6 of 6

35. Huxley RR, Woodward M (2011) Cigarette smoking as a risk factor for coronary heart disease in women compared with men: a systematic review and meta-analysis of prospective cohort studies. Lancet 378: 1297-1305.

36. Dean J, Cruz S, Mehta P, Merz CNB (2015) Coronary microvascular dysfunction: sex-specific risk, diagnosis, and therapy. Nat Rev Cardiol 12 : 406-414.

37. Sucato V, Evola S, Novo G, Novo S (2013) Diagnosis of coronary microvascular dysfunction in diabetic patients with cardiac syndrome $X$ comparison by current methods. Recenti Prog Med 104: 63-68.

38. Carli MFD, Janisse J, Grunberger G, Ager J (2003) Role of chronic hyperglycemia in the pathogenesis of coronary microvascular dysfunction in diabetes. J Am Coll Cardiol 41: 2387-2393.

39. Liao KP, Huang J, He Z, Cremone G, Lam E, et al. (2019) Coronary microvascular dysfunction in rheumatoid arthritis compared to diabetes mellitus and association with all-cause mortality. Arthritis Care Res.

40. Ormseth MJ, Oeser AM, Cunningham A, Bian A, Shintani A, et al. (2014) Reversing vascular dysfunction in rheumatoid arthritis: improved augmentation index but not endothelial peroxisome proliferator-activated receptor gamma agonist therapy. Arthritis rheumatology 66: 2331-2338.

41. Turiel M, Tomasoni L, Sitia S, Cicala S, Gianturco L, et al. (2010) Effects of long-term disease-modifying antirheumatic drugs on endothelial function in patients with early rheumatoid arthritis. Cardiovasc Ther 28: 53-64.

42. Ishimori ML, Martin R, Berman DS, Goykhman P, Shaw L, et al. (2011) Myocardial ischemia in the absence of obstructive coronary artery disease in systemic lupus erythematosus. JACC Cardiovasc Imaging 4: 27-33.

43. Shaw LJ, Bairey Merz CN, Pepine CJ, Reis SE, Bittner V, et al. (2006) Insights from the NHLBI-Sponspored Women's Ischemia Syndrome Evaluation (WISE) Study: Part I: gender differences in traditional and novel risk factors, symptom evaluation, and gender-optimized diagnostic strategies. J Am Coll Cardiol 47: S4-S20.

44. Wessel TR, Arant CB, McGorray SP, Sharaf BL, Reis SE, et al. (2007) Coronary microvascular reactivity is only partially predicted by atherosclerosis risk factors or coronary artery disease in women evaluated for suspected ischemia: results from the NHLBI Women's Ischemia Syndrome Evaluation (WISE). Clin Cardiol 30: 69-74.

45. Kaski JC (2006) Cardiac syndrome $X$ in women: the role of oestrogen deficiency. Heart 92: iii5-iii9.

46. Moreau $P$, d'Uscio L, Lüscher TF (1998) Structure and reactivity of small arteries in aging. Cardiovasc Res 37: 247-253.

47. Gulati M, Cooper-DeHoff RM, McClure C, Johnson BD, Shaw LJ, et al. (2009) Adverse cardiovascular outcomes in women with nonobstructive coronary artery disease: a report from the Women's Ischemia Syndrome Evaluation Study and the St James Women Take Heart Project. Arch Intern Med 169: 843-850.

48. Collins P, Maas A, Prasad M, Schierbeck L, Lerman A, et al. (2020) Endothelial vascular function as a surrogate of vascular risk and aging in women. Mayo Clin Prod 95: 541-553.

49. Bechlioulis A, Kalantaridou SN, Naka KK, Chatzikyriakidou A, Calis KA, et al. (2010) Endothelial function, but not carotid intima-media thickness, is affected early in menopause and is associated with severity of hot flushes. J Clin Endocrinol Metab 95: 1199-1206

50. Chambers JC, Fusi L, Malik IS, Haskard DO, De SM, et al. (2001) Association of maternal endothelial dysfunction with preeclampsia. JAMA 285: 16071612.

51. Hayman R, Warren A, Brockelsby J, Johnson I, Baker P, et al. (2000) Plasma from women with pre-eclampsia induces an in vitro alteration in the endothelium-dependent behaviour of myometrial resistance arteries. BJOG 107: 108-115.

52. Lau SY, Guild SJ, Barrett CJ, Chen Q, McCowan L, et al. (2013) Tumor necrosis factor-alpha, interleukin-6, and interleukin-10 levels are altered in preeclampsia: a systematic review and meta-analysis. Am J ReprodImmunol 70: 412-427.

53. Boeldt DS, Bird IM (2017) Vascular adaptation in pregnancy and endothelial dysfunction in preeclampsia. J Endocrinol 232: R27-R44.

54. Visser S, Hermes W, Ket JC (2014) Systematic review and metanalysis on non-classic cardiovascular biomarkers after hypertensive pregnancy disorders. Am J Obstet Gynecol 211: 373-379.

55. Bellamy L, Casas JP, Hingorani AD, Williams D (2009) Type 2 diabetes mellitus after gestational diabetes: a systematic review and meta-analysis. Lancet 373: 1773-1779.
56. Mathews L, Iantorno M, Schar M, Bonanno G, Gerstenblith G, et al. (2017) Coronary endothelial function is better in healthy premenopausal women than in healthy older postmenopausal women and men. PLOS ONE 12: e0186448.

57. Atsma F, Bartelink ML, Grobbee DE, van der Schouw YT (2006) Postmenopausal status and early menopause as independent risk factors for cardiovascular disease: a meta-analysis. Menopause 13: 265-279.

58. Kok HS, van Asselt KM, van der Schouw YT (2006) Heart disease risk determines menopausal age rather than the reverse. J Am CollCardiol 47: 1976-1983

59. Matthews KA, Crawford SL, Chae CU (2009) Are changes in cardiovascular disease risk factors in midlife women due to chronological aging or to the menopausal transition? J Am CollCardiol 54: 2366-2373.

60. Mosca L, Benjamin EJ, Berra K, Bezanson JL, Dolor RJ, et al. (2011) Effectiveness-based guidelines for the prevention of cardiovascular disease in women-2011 update: a guideline from the American Heart Association. Circulation 123: 1243-1262. 